\title{
INVESTIGACIONES
}

\section{Desenganche escolar-cultura organizativa: implicaciones mutuas en las Aulas Ocupacionales*}

\author{
School Disengagement-organizative culture: \\ mutual implications in the Aulas Ocupacionales
}

$M^{a}$ Teresa González González ${ }^{a}, M^{a}$ Trinidad Cutanda López ${ }^{a}$

${ }^{\text {a }}$ Departamento de Didáctica y Organización Escolar. Facultad de Educación. Universidad de Murcia, España. mtgg@um.es, lopez.cutanda@um.es

\section{RESUMEN}

Se presentan y analizan en este artículo algunos datos derivados de una Tesis Doctoral sobre Aulas Ocupacionales (AO) en la Región de Murcia (España), una medida destinada a estudiantes con comportamientos absentistas y disruptivos en la etapa de Educación Secundaria Obligatoria. En particular, aquellos que evidencian que en los procesos de desenganche de este alumnado influye la propia experiencia escolar que se les ofrece y que está condicionada por la cultura organizativa de los centros escolares y sus aulas. Supuestos básicos, creencias y valores mantenidos por docentes y otros profesionales del AO ponen de manifiesto la pervivencia de una lógica de déficit reflejada en su funcionamiento habitual "organizativo y pedagógico". Esto constituye una barrera sustancial para hacer del AO un espacio rico de atención educativa, aminorar la desafección de sus estudiantes con sus aprendizajes y su alejamiento de lo escolar, y empujarlos a persistir e implicarse con su formación.

Palabras claves: Absentismo escolar, calidad de la educación, Centro de Enseñanza Secundaria, Educación Básica.

\section{ABSTRACT}

This article presents and analyzes some data derived from a doctoral thesis about Aulas Ocupacionales (AO) in the Region of Murcia (Spain), which represent a measure aimed at students with a history of truancy and bad behavior in the Secondary Basic Education stage. In particular, those that show that in the disengagement processes of this student influences the own school experience offered and reflected in the organizational culture of the schools and their classrooms. Basic assumptions, beliefs and values kept by the teachers and other professionals of the AO reveal the continued existence of the logic of the deficit, reflected in the daily operation "organizational and pedagogical". This constitutes a substantial barrier to turn the AO into an environment rich in educational attention for students disengaged in their learnings, lower their disaffection and distance from the academic environment and push them to persist and involve themselves in their training.

Key words: Basic education, quality education, Secondary School, Truancy.

* El artículo se deriva de la Tesis Doctoral titulada "La implicación del alumnado absentista y en riesgo de abandono en su propio aprendizaje y el papel de la formación, el compromiso y el trabajo en el aula de los docentes. El caso de las Aulas Ocupacionales de la Región de Murcia”, financiada por el Ministerio de Economía y Competitividad (Ayuda Pre-Doctoral BES-2013-036943). 


\section{INTRODUCCIÓN}

En este artículo se presenta y analiza información relacionada con el funcionamiento de una medida educativa -Aula Ocupacional (AO)- expresamente diseñada y desarrollada en la Comunidad Autónoma de la Región de Murcia (CARM) para alumnado con historial disruptivo y de absentismo en los primeros cursos de la Educación Secundaria Obligatoria (15 años). Aunque el foco de atención del análisis se sitúa en las decisiones tomadas respecto a esta medida de "Atención a la Diversidad" y acciones realizadas en su discurrir cotidiano, se pondrá la mirada expresamente en los supuestos, valores, creencias, principios que subyacen o dan sentido a tales decisiones y actuaciones. El propósito es ilustrar cómo no siempre ésos se corresponden o alinean con los formalmente declarados en normativas y documentos oficiales, manteniéndose prácticas y actuaciones organizativas y pedagógicas que están asentadas en supuestos y creencias ya profundamente arraigados y difíciles de modificar.

El texto se estructura en 4 apartados. Tras la introducción, se plantea sucintamente el marco teórico de partida articulado sobre tres ideas básicas: a) la investigación sobre procesos de desenganche escolar aporta coordenadas teóricas y conceptuales relevantes para abordar el estudio de problemas de absentismo y comportamentales, en cuanto que manifestación de tales procesos; b) los propios centros escolares con sus modos de hacer y funcionar pueden constituir un factor de riesgo, entre otros, de tal desenganche; c) la "cultura" organizativa -creencias, valores, normas implícitas, supuestos básicos -que se ha ido configurando en centros y aulas influye y sostiene modos de hacer que pueden facilitar, o al contrario, contrarrestar el desenganche de estudiantes. Asimismo, se acota el concepto de "cultura" organizativa y se ofrece una panorámica de lo investigado sobre el tema en relación con procesos de enganche-desenganche. Tras esta sucinta revisión, en el apartado tres se describe brevemente la investigación -diseño metodológico, recogida de información y análisis de la misma- de la que derivan los datos que se presentan en el cuarto apartado, para finalizar con algunas reflexiones y conclusiones derivadas de los mismos.

\section{MARCO TEÓRICO}

\subsection{IMPLICACIÓNY DESAFECCIÓN DELALUMNADO: LA CONTRIBUCIÓN DEL CENTRO ESCOLAR}

Lo que ocurre dentro de los centros escolares puede posibilitar y favorecer tanto el enganche del alumnado como contribuir a su progresivo desenganche y, finalmente, abandono, como así ha ido constatando investigación relativamente reciente sobre el tema que advierte de la importancia del contexto escolar en el que transcurre la trayectoria educativa del alumnado, de cómo interaccionan con ese contexto y responden -en términos de mayor o menor engagement- a lo que ocurre en él (Ariastimuñi y Parodi, 2017; González, 2015; Friensen, 2010; Hynes, 2014; LAB, 2001; McMahon, Munns Smyth \& Zygnier, 2012; Pinya Pomar y Salvà-Mut, 2017; Salvà-Mut, Oliver-Trobat y Comas-Forgas, 2014; Tarabini et al., 2015; Taylor \& Parsons, 2011; Willms, Friesen \& Milton, 2009). Esas y otras investigaciones sustentan que, así como pueden existir factores de riesgo de desenganche ligados a las características del propio estudiante y sus entornos socioculturales, económicos 
y familiares, también los centros escolares -a través de sus dinámicas, y prácticas curriculares y organizativas cotidianas- pueden constituir un entorno de riesgo (Escudero y González, 2013).

No se trata de traspasar la "culpa" a centros y docentes, pues el problema es multifactorial y mutidimensional: los centros escolares no funcionan al margen del sistema educativo del que forman parte y sus políticas educativas ni, tampoco, éstas son independientes de las estructuras sociales, políticas, culturales y económicas imperantes (Vázquez Recio et al., 2018; Vázquez Recio y López Gil, 2018). No se puede pasar por alto la influencia de esas amplias coordenadas en su configuración y prácticas cotidianas. Pero, en cualquier caso, lo que ocurre en cada centro, importa: ciertos estudiantes se alejan, pierden interés y se desapegan de lo escolar y de los aprendizajes que se les están tratando de posibilitar. Como advertía Smyth (2006), políticas basadas únicamente en nociones de responsabilidad individual necesitan ser equilibradas con el análisis y comprensión de cómo las propias instituciones fracasan con los jóvenes.

El desenganche de estudiantes expresa el desajuste que puede existir entre ellos y un centro escolar que les plantea unas demandas y exigencias, les brinda o no apoyos y acogidas, les ofrece una experiencia más o menos cercana o lejana, más o menos relevante o no-significativa, inclusiva o excluyente. Son múltiples las facetas del centro escolar que pueden influir tanto en sentido positivo como negativo, sobre el enganche del alumnado y su rendimiento -por ejemplo, sus estructuras de coordinación y cómo se utilizan; clima relacional y convivencia; oferta curricular y su despliegue en las aulas; desarrollo de tutorías y orientación a estudiantes; liderazgo; relación con familias...-. A todas, subyace y confiere sentido una determinada "cultura organizativa" (Brady, 2005), cuya importancia es subrayada por Ladson-Billings (2006) cuando afirma:

(...) miramos a los estudiantes como individualmente responsables de su éxito en el centro escolar. Carecemos de una comprensión profunda de cómo factores individuales, familiares, de la comunidad, escolares y sociales interactúan para generar fracaso escolar de algunos estudiantes. Es mucho más fácil explicar el fracaso de los alumnos mirando a algo interno a ellos que endémico a eso que denominamos cultura escolar (p. 106).

\subsection{CULTURA ORGANIZATIVA}

No existe una acotación conceptual unívoca y unánimemente aceptada de "Cultura Organizativa”. Hay múltiples definiciones (Kotter, 1996; López Yáñez, 2004; Sarasola, 2004; Schein,1992; Van Houtte, 2005), tipologías (Connolly, James \& Beales, 2011) así como diferentes análisis sobre las muchas culturas en conflicto dentro de la escuela (Brown, 2004). Se entenderá aquí que dicha expresión alude al conjunto de supuestos, creencias, valores, normas implícitas que subyacen y apuntalan lo que se hace en el centro escolar, sus rutinas pedagógicas y organizativas. Con frecuencia permanece implícita, pero es una dimensión organizativa que "impregna" todas las facetas del centro escolar y su funcionamiento como tal. Esto es, el cómo se interpreta, comprende y actúa en sus diversos ámbitos (pedagógico, organizativo, socio-relacional, etc.) no es sino la expresión de creencias, valores, supuestos implícitos sobre el modo más deseable y adecuado de hacer las cosas. 
Aunque suelen coexistir subculturas ligadas a grupos particulares o unidades organizativas, la cultura del centro escolar (quizás definida por su propia fragmentación en subculturas) determina, entre otros: la noción más o menos compartida del sentido y propósitos del centro y la educación que ha de ofrecer; el cómo transcurren los procesos de toma de decisión organizativa; el currículo que se desarrolla; el clima relacional, de convivencia y de aprendizaje; el modo y sentido con el que se llevan a cabo procesos de planificación, liderazgo, gestión, evaluación, coordinación, etc. (González, 2003, 2005). Shafritz \& Ott, (2001) expresan esta idea señalando que "la mayoría de las conductas y las decisiones organizativas están, en efecto, predeterminadas por los patrones de supuestos básicos que sostienen los miembros" (p. 361-362). Subrayan así que éstos influyen en las conductas y modos de hacer de sus miembros tanto o más que las reglas y estructuras formales del centro escolar.

\subsubsection{Cultura organizativa: su influencia en el enganche/desenganche del alumnado}

La cultura de los centros escolares no se construye únicamente desde y a partir del propio centro: factores y elementos en el ámbito de políticas educativas, sociales, económicas, culturales, entre otras (Escudero, 2016; Vázquez Recio et al., 2018), articulan discursos dominantes que, a la larga, la conforman y determinan. Es clara, por ejemplo, la preeminencia e influencia que sobre el particular ha tenido un "discurso de déficit y culpa" (McInerney, 2006) que ha tendido a patologizar a los estudiantes, culparlos a ellos, sus familias, barrios y/o entornos culturales, de su desafección por los aprendizajes escolares. Se da por sentado que el riesgo de desenganche está localizado en las limitaciones del individuo, su familia y contexto, no en las prácticas estructurales e institucionales y relaciones inherentes de poder, que no se cuestionan. Ese discurso de déficit influye en las culturas de los centros y, en general, en el pensamiento y concepciones mantenidas en ellos acerca de las actuaciones con el alumnado. Como apuntó Zyngier (2005) la visión de déficit sienta la base para asumir que es necesario el dirigismo, pues se iguala la noción de enganche con conformidad del estudiante a las reglas y a participar en actividades establecidas, determinadas y conducidas por adultos.

Múltiples trabajos de investigación constatan la incidencia de la cultura escolar tanto en un sentido positivo (facilitar) o negativo (impedir), en el grado de implicación del estudiante con sus aprendizajes y permanencia en el centro. A título ilustrativo, se recogen seguidamente algunas referencias:

- Un ambiente escolar alienante y una cultura que no es favorable a las necesidades de los estudiantes son factores significativos que inciden en procesos de desenganche escolar (Dwyer, Osher \& Warger, 1998).

- Las culturas escolares no son independientes del sentido que los estudiantes den a su experiencia en el centro escolar: no es lo mismo que en él se trate a los alumnos de modo inclusivo, se sea indiferente al tema o, incluso, se sostenga un planteamiento inhóspito y excluyente; en cada caso, las políticas, prácticas y acciones escolares, serán diferentes (Smyth \& Hattam, 2002).

- Los supuestos mantenidos acerca del propósito de la educación y de los papeles de docentes y estudiantes impactarán las creencias sobre el enganche del alumnado (Vibert \& Shields, 2003). 
- Para la gran mayoría de jóvenes, abandonar la escuela está influido por la naturaleza insatisfactoria de la cultura escolar. Una cultura que no apoya, sin interés, no retadora, autoritaria, punitiva, anquilosada, inflexible, violenta- es un factor de riesgo (Clark, Borj, Calleja, Chircop \& Portelli, 2005).

- La cultura escolar tiene una influencia constante sobre los estudiantes. Es más probable que quienes asisten a centros escolares que exhiben propósito claro, equidad para todos, apoyo y atención individual, que facilitan el que todos puedan experimentar el éxito y en los que hay atención y cuidado, desarrollen un sentido de pertenencia e identificación con la escuela y sus valores (Russell, Ainley \& Frydenberg, 2005).

- Culturas escolares asentadas en la violencia, acoso, discriminación, abuso, racismo, tratamiento inadecuado por parte de los compañeros de clase, incapacidad para gestionar la jornada escolar inciden negativamente en el enganche de los estudiantes (Ferguson et al., 2005).

- Una cultura escolar en la que se prioriza habilidades y excelencia académica pone en desventaja a muchos jóvenes y provoca que se desconecten del aprendizaje (Bielby, Judkings, O’Donnell \& McCrone, 2012).

- Una cultura escolar positiva, caracterizada por un ambiente escolar seguro, relaciones positivas docente-estudiante, un enfoque más abierto e inclusivo para implicar a padres de estatus socio-cultural más bajo y/o de background inmigrante o de minoría étnica, un enfoque más holístico hacia las necesidades de los jóvenes, etc.; pueden ser factores protectores cruciales frente a procesos de desenganche escolar y abandono temprano (Clycq et al., 2017, p. 18).

Aportaciones como las anteriores dejan entrever que valores, creencias y supuestos básicos orientan e impregnan el discurrir cotidiano de la organización educativa, y repercuten en los niveles de desenganche. Que tal influencia pueda producirse positiva o negativamente es indicativo de que no solo importa compartir valores, creencias y supuestos básicos. También, cuáles son, sobre qué versan, qué propuestas pedagógicas anidan. Supuestos y valores, aunque compartidos y asumidos, pueden resultar disfuncionales, justificar prácticas organizativas y curriculares que solo beneficien a algunos, sean injustas o no-equitativas. En otro sentido, sin detenernos en esto, también múltiples aportaciones (Ferguson et al., 2005; Smyth, 2006, 2012: Wilson, Stemp \& McGinty, 2011) subrayan cómo las culturas de algunos estudiantes pueden entrar en conflicto o estar muy alejadas de la cultura escolar convencional, y el posible desencuentro entre ambas alimenta el desapego y desenganche.

\subsubsection{Aminorar la desafección escolar. El difícil trayecto de alterar culturas}

Los centros desempeñan un papel importante en promover el desenganche escolar: culturas asentadas en planteamientos de déficit, supuestos y valores que sacralizan el conocimiento académico, la enseñanza "frontal" dirigida por el docente, modelos de disciplina punitivos que valoran sobremanera la sumisión del alumnado (Thomson \& Pennachia, 2016). Revertir situaciones de absentismo, desafección y abandono, pasa, entre otros, por mejorar lo que ocurre en ellos; pero serán mejoras superficiales y pasajeras si no alteran la cultura subyacente. Como argumentó en su momento Schein (1992), para cambiar el modo en que funciona una organización, el cambio ha de ocurrir en la estructura de creencias básicas de 
los miembros de modo que, si no se saca a la luz y cuestionan los supuestos básicos, y los individuos no se implican en procesos de transformación cognitiva (p. 19), será difícil que los cambios en cómo se hacen las cosas sean sostenibles y duraderos.

Alterar esa "gramática básica" (Tyack \& Cuban, 2000) que determina la vida escolar, transformar mentalidades y desaprender cosas dadas por ciertas, es particularmente relevante en el tema que nos ocupa. La propia European Comission (2015) apunta que: "es necesario promover una cultura escolar positiva, trabajo en equipo y prácticas colaborativas en la comunidad escolar (...) para asegurar el éxito educativo y prevenir el abandono escolar temprano" (p. 5). Otras propuestas y planteamientos sobre el particular, ya sea más centrados en aspectos educativos o curriculares, como en otros más sociales, así lo corroboran. Desde una mirada educativa Azevedo (2014) reclama la necesidad de:

Generar otros modos de acción pedagógica y de organización escolar y proporcionar en las escuelas otras propuestas socioeducativas, pues la violencia social ya alcanza a obligar a todos los ciudadanos que coman el mismo 'plato' y 'angélicamente' esperamos que todos coman todo, al mismo tiempo, con la misma voluntad y con el mismo grado de satisfacción durante doce años seguidos. ¡Esperamos que los elefantes vuelen! (pp. 2-3).

Otros autores aluden a una cultura que apuntale pedagogías de re-enganche en la que el punto de partida no sea qué currículo y enseñanza ofrece el centro sino, qué requieren los estudiantes considerando sus capacidades, ritmos, intereses, preocupaciones, esperanzas, para a partir de ahí trabajar el currículo (Athew, Bland, Carrinton \& Cavanagh, 2007; Brown, 2004). En líneas similares, pero poniendo el énfasis en aspectos sociales, más que pedagógicos, se reclama la necesidad de cultivar culturas que apoyen la seguridad, la expresión individual, el tratamiento justo, la voz del alumnado (Shoda \& Guglielmi, 2009), o culturas de 'cuidado' flexibilidad, comprensión, bienvenida, reconocimiento y atención a todos (Tilleczek et al., 2011), respetuosa, inclusiva que anime a los estudiantes a incorporar sus propias identidades y las de sus familias en sus experiencias de aprendizaje (Shields, 2003). Una idea que sobrevuela en las investigaciones e informes consultados es la insistencia en la necesidad de repensar y modificar tanto el pensamiento de déficit, como la visión que lo acompaña del desenganche como problema individual y responsabilidad del propio estudiante.

\section{METODOLOGÍA}

Los datos presentados proceden de una investigación sobre Aulas Ocupacionales en la Región de Murcia (España) con el propósito de indagar sobre las claves políticas, institucionales, organizativas y pedagógicas en su génesis, diseño y desarrollo, y los resultados logrados (de aprendizaje y re-enganche de sus estudiantes). Se posicionó en un Enfoque de Métodos Mixtos de Investigación -Cualitativo-cuantitativo- (Creswell, 2015) en consonancia con la complejidad de la problemática del desenganche escolar que requiere de un análisis complejo de los múltiples planos, condiciones, factores y dimensiones que de forma interrelacionada y dinámica intervienen en el mismo. Una postura respaldada tanto por estudiosos del tema como en particular por investigadores e investigadoras cuyo foco de análisis han sido distintos programas y medidas destinadas a estudiantes en situación de vulnerabilidad (González, 2015; Escudero et al., 2013; Fredericks et al., 2004; McMahon 
\& Zyngier, 2009; Salvà-Mut et al., 2014; Taylor \& Paterson, 2011). En concreto, se optó por un Diseño de Métodos Mixtos Anidado Convergente Multinivel de Método Dominante Cualitativo (Hernández-Samperi, Fernández y Baptista, 2014). El método descriptivo se anidó en el fenomenológico -prioritario- posibilitando junto al análisis deductivo, una mayor comprensión desde la significación de sus participantes en múltiples niveles de análisis considerados: políticas educativas -macro-, centro escolar -meso- y aula -micro-. Todos los participantes seleccionados pertenecen a un mismo universo que fue abordado íntegramente. La población coincidió así con la muestra no siendo necesario realizar ningún proceso de selección muestral (Arias, 2012). El conjunto de casos estudiados corresponde a las siete AO vigentes en la CARM durante el curso escolar 2016-2017, sus estudiantes, docentes y otros profesionales implicados en ellas. Los datos cuantitativos y cualitativos se recabaron en una sola fase empleando diversos instrumentos. Para recoger la información cuantitativa se diseñaron y validaron dos cuestionarios dirigidos a estudiantes y docentes. La de carácter cualitativo se recabó mediante entrevistas, análisis legislativo y documental y observaciones de aula. La información se trianguló e integró, tanto en su recogida como en la interpretación de los resultados -metainferencias- acorde con el planteamiento general de un diseño concurrente (Teddlie \& Tashakkori, 2010), siendo analizada por separado. Se garantizó la calidad y el rigor de la investigación en todas las etapas de su desarrollo atendiendo a los criterios esenciales delimitados por Hernández-Sampieri et al. (2014): calidad en el diseño; rigor interpretativo y legitimidad.

En el presente artículo se presenta y comenta únicamente la información cualitativa recabada de los distintos profesionales implicados en el $\mathrm{AO}$ que nos ha permitido evidenciar cuál es la influencia de la cultura organizativa de los centros escolares vinculados a tales Aulas en su funcionamiento y resultados: Administración Regional $(\mathrm{N}=1)$; Administración local (6 técnicos del programa de absentismo de Ayuntamientos y 3 educadores sociales contratados por éstos destinados al AO); Equipos Directivos ( $\mathrm{N}=7)$; Departamentos de Orientación ( $=$ 12); Equipo Docente $(\mathrm{N}=17)$. Las siete $\mathrm{AO}$ quedaron así representadas en todos los colectivos implicados con el programa. Tras contactar y obtener los permisos de Administración, centros y entrevistados, y siempre salvaguardando el anonimato, confidencialidad, ética y rigor de investigación, se accedió al campo durante septiembre 2016-junio 2017. La información que aquí concierne se obtuvo mediante entrevistas semi-estructuradas adaptadas a los diversos colectivos mencionados cuyas guías se validaron mediante juicio de expertos. En la única dirigida al representante de la Administración fueron temas de interés: la conformación definitiva del mapa del $\mathrm{AO}$ en el período de estudio; motivos o ejes sobre los que se sustenta la normativa y valoraciones sobre éstas y sus resultados. En las restantes: roles y funciones de los profesionales vinculados al AO; perfil del alumnado; criterios de adscripción, capacitación e implicación con su labor del profesorado; y valoración global sobre las mismas. Se realizó un total de 28 entrevistas (once grupales) oscilando entre 30-190 minutos. El procesamiento y análisis de datos siguió un modelo deductivo-inductivo de análisis de contenido (Miles, Huberman y Saldaña, 2014), en cuatro fases: a) Pre-análisis. Transcripción de entrevistas; archivo, reorganización y relectura global. b) Definición de Unidades temáticas de análisis de libre flujo. c) Codificación abierta y establecimiento de reglas de análisis y códigos de clasificación mediante diversas técnicas de escrutinio, y de procesamiento inductivo de comparación constante entre unidades de análisis emergentes. Se identificaron 150 códigos. d) Desarrollo de Categorías conceptuales. Emergieron 11 categorías centrales en los tres planos de indagación contemplados (Tabla 1). Se realizó con el apoyo del software Atlas ti V8. 
Tabla 1. Categorías y subcategorías derivadas del procesamiento de análisis de contenido cualitativo en los niveles estudiados (macro-meso y micro)

\begin{tabular}{|c|c|c|}
\hline Nivel de análisis & Categorías de análisis & Subcategorías de análisis \\
\hline \multirow[t]{3}{*}{$\begin{array}{l}\text { Contexto socio- } \\
\text { político-administrativo } \\
\text { y educativo (macro) }\end{array}$} & $\begin{array}{l}\text { Necesidad y justificación (de } \\
\text { las AO en la Región de Murcia) }\end{array}$ & $\begin{array}{ll}- & \text { Origen } \\
- & \text { Cambios } \\
- & \text { Normativa } \\
\end{array}$ \\
\hline & $\begin{array}{l}\text { Diseño oficial (de las AO } \\
\text { durante su estudio y principales } \\
\text { modificaciones respecto a su } \\
\text { regulación inicial) }\end{array}$ & $\begin{array}{ll}- & \text { Ordenación } \\
\text { - } & \text { Oferta } \\
\text { - } & \text { Activación } \\
\text { - } & \text { Evaluación } \\
\end{array}$ \\
\hline & $\begin{array}{l}\text { Mapa de distribución e } \\
\text { implantación (en la red de } \\
\text { Ayuntamientos y centros y } \\
\text { motivos para su implantación) }\end{array}$ & $\begin{array}{ll}- & \text { Ayuntamientos } \\
- & \text { Centros de Educación Secundaria }\end{array}$ \\
\hline \multirow[t]{5}{*}{$\begin{array}{l}\text { Programa en acción } \\
\text { (niveles meso y micro) }\end{array}$} & Alumnado & $\begin{array}{ll}\text { - } & \text { Características socio-familiares y } \\
& \text { personales } \\
\text { - } & \text { Trayectoria escolar } \\
\text { - } & \text { Derivación al AO } \\
\text { - } & \text { Visión y expectativas } \\
& \text { institucionales y docentes }\end{array}$ \\
\hline & Profesorado & $\begin{array}{ll}\text { - } & \text { Adscripción al AO } \\
\text { - } & \text { Preparación previa y desarrollo } \\
& \text { profesional } \\
\text { - } & \text { Implicación (Teacher Engagement) } \\
\text { - } & \text { Visión institucional y del } \\
& \text { alumnado }\end{array}$ \\
\hline & Condiciones organizativas & $\begin{array}{ll}- & \text { Estructura } \\
- & \text { Cultura organizativa } \\
- & \text { Relaciones } \\
- & \text { Procesos } \\
- & \text { Entorno } \\
\end{array}$ \\
\hline & Núcleo Pedagógico & $\begin{array}{ll}\text { - } & \text { Currículo } \\
\text { - } & \text { Enseñanza-aprendizaje }\end{array}$ \\
\hline & $\begin{array}{l}\text { Valoración de las AO (por los } \\
\text { distintos implicados en ellas) }\end{array}$ & $\begin{array}{ll}\text { - } & \text { Como programa } \\
\text { - } & \text { Sobre la experiencia } \\
\text { - } & \text { Propuestas de mejora } \\
\text { - } & \text { Expectativas de futuro }\end{array}$ \\
\hline \multirow[t]{3}{*}{ Resultados } & \multicolumn{2}{|l|}{ Aprendizajes logrados } \\
\hline & Student Engagement & $\begin{array}{ll}- & \text { Conductual } \\
- & \text { Afectivo } \\
- & \text { Cognitivo } \\
\end{array}$ \\
\hline & $\begin{array}{l}\text { Opciones (tras finalizar el } \\
\text { programa) }\end{array}$ & $\begin{array}{ll}\text { - } & \text { Académicas } \\
\text { - } & \text { Profesionales }\end{array}$ \\
\hline
\end{tabular}

Fuente. Elaboración propia. 


\section{RESULTADOS Y DISCUSIÓN}

Se presentan y comentan algunas de las creencias y supuestos respecto al AO y su alumnado, que cabe derivar el análisis de las entrevistas a los profesionales implicados.

\subsection{LOS ESTUDIANTES DEL AO: VISIÓN DE DÉFICIT}

Es manifiesta la predominancia y vigencia de un "pensamiento de déficit" que subyace a prácticamente todas las decisiones que se han ido tomando sobre esta medida para alumnado absentista (Tabla 2). Las propias administraciones (autonómica y local) sostienen discursos plagados de supuestos que condicionan y refuerzan los ya comúnmente cultivados en los Institutos, relacionados con esa visión de que son los estudiantes "los que tienen la culpa".

Tabla 2. El alumnado y "sus déficits"

\begin{tabular}{|c|c|}
\hline $\begin{array}{l}\text { Administración } \\
\text { Autonómica y Local } \\
\text { (ADM) }\end{array}$ & $\begin{array}{l}\text { - } \quad \text { Problemáticos. } \\
\text { - } \quad \text { No favorecen la imagen y resultados del centro. } \\
\text { - } \quad \text { Lo rechaza todo, ...no va a interiorizar la jerarquía del Instituto ni } \\
\text { acatar las normas... } \\
\text { - } \quad \text { En el IES: volvemos al modelo de listos y tontos. }\end{array}$ \\
\hline $\begin{array}{l}\text { Equipo Directivo } \\
\text { (ED) }\end{array}$ & $\begin{array}{l}\text { - ...En el centro no podía o no era capaz de integrarse. (...) su manera } \\
\text { de expresarse, situarse, relacionarse, es totalmente inadecuada (...) } \\
\text { están perdiendo el tiempo... no aprovechan las oportunidades que le } \\
\text { ofrecen. } \\
\text { - } \\
\text { Tienen fobia al Instituto. Rasgos... que impiden estar en un centro } \\
\text { totalmente normalizado. } \\
\text { - ..Al borde de la Fiscalía de Menores. } \\
\text { - Dificultades curriculares todas... el propio alumno trae vicios y } \\
\text { errores. }\end{array}$ \\
\hline Docentes & $\begin{array}{ll}\text { - } & \text { Sin: hábitos, costumbres, paciencia, capacidad de atención, } \\
\text { - } & \text { motivación... } \\
\text { - } & \text { Consumidomáticas familiares; relaciones traumáticas. } \\
\text { - } & \text { Vistos en el Instituto: mataos, en el fondo del saco, ogros, gente } \\
& \text { mala... } \\
\text { - } & \text { Conflictivos, no enraízan (...) hay que aceptar que el fracaso una } \\
& \text { opción más. } \\
\text { - Intelectualmente no dan más. }\end{array}$ \\
\hline $\begin{array}{l}\text { Depto. Orientación } \\
\text { (DO) }\end{array}$ & $\begin{array}{l}\text { - } \quad \begin{array}{l}\text { Familias: idea de "mi hijo no va a ir (AO) porque va toda la } \\
\text { chusma". }\end{array} \\
\text { - } \quad \text { Habría que seleccionarlos mucho más; el perfil es muy complejo. } \\
\text { - } \quad \text { Alumno: no el enemigo, sí el contrario. } \\
\text { - Forma parte de la cultura en el ámbito educativo, lo dicho para el } \\
\text { AO, vale para PMAR, la antigua diversificación curricular, la FP } \\
\text { Básica, para todo: ahí van los tontos. }\end{array}$ \\
\hline
\end{tabular}

Fuente. Elaboración propia. 


\subsection{EL AO MEJOR FUERA DEL IES}

Es la Administración la que sustenta y decide, con el beneplácito e, incluso presión por parte de los IES, que, aunque sean alumnos que están en escolaridad obligatoria, no tienen que estar físicamente en los centros (Tabla 3).

Tabla 3. Ubicación externa del AO

\begin{tabular}{|c|c|}
\hline ADM & $\begin{array}{l}\text { - Reticencias del IES a aceptar a un gran número de alumnos que harían bajar en } \\
\text { el escalafón del ranquin de centros. } \\
\text { - } \quad \text { Los Institutos ponen el grito en el cielo al derivar al AO alumnos con expediente } \\
\text { disciplinario. } \\
\text { - } \quad \text { Vistos como terroristas... han de estar lo más alejados posible del IES. } \\
\text { - Desde fuera, así tiene que funcionar y la Consejería lo consideró oportuno. }\end{array}$ \\
\hline ED & $\begin{array}{l}\text { - Rechazo a dar clase en el AO: Docentes no dispuestos a incluir dentro del } \\
\text { Instituto a ese alumnado } \\
\text { - Es un error empeñarse en que todo el mundo tiene que estar en el IES. No todos } \\
\text { tienen que tener una respuesta ... permitirles una flexibilidad que en el IES no } \\
\text { pueden tener. } \\
\text { - No es buena publicidad para el IES: ha de ofrecer ofertas de calidad } \\
\text { - En el IES se presta más atención al alumnado con necesidades educativas } \\
\text { especiales, (...) que a ese otro tipo de alumnado. } \\
\text {... allí estarán mejor...no hace falta que tengan expediente de absentismo. }\end{array}$ \\
\hline Docentes & $\begin{array}{l}\text { - Con el AO (...) todos los chicos que están dando problemas, nos los quitamos de } \\
\text { en medio. } \\
\text { ¿Cuál es el beneficio del AO? Descongestionas las aulas de alumnos que } \\
\text { molestan, no hacen nada, no tienen interés. }\end{array}$ \\
\hline DO & $\begin{array}{l}\text { - ...si hubieran estado aquí (IES), habrían estado expulsados todo el año. } \\
\text { - El profesorado valora muy bien que se derive al AO a alumnos difíciles para los } \\
\text { que el IES no está adaptado. } \\
\text { El director fue firme: si esto va a suponer un perjuicio mayor que un beneficio, } \\
\text { no vale la pena. }\end{array}$ \\
\hline
\end{tabular}

Fuente. Elaboración propia.

A la luz de los argumentos recogidos en las Tablas anteriores, dos son los razonamientos que justifican la decisión de localizar el AO fuera del IES:

1) Las características del propio alumnado: se reitera por los representantes de ambas administraciones y equipos directivos que se trata de estudiantes problemáticos, con rechazo al IES, incapaces de integrarse, con un perfil al que el Instituto no puede dar respuesta. 2) La prioridad de los IES es cultivar la calidad y la excelencia. Miembros de equipos directivos, en general, concuerdan. Incluso, algunos Institutos que tradicionalmente se habían "responsabilizado" del Aula, rechazan la posibilidad de emplazarla en los IES. Entienden que ello, en términos de esfuerzos y resultados obtenidos por el centro, supondría "más perjuicios que beneficios". 
Ambos argumentos se entrelazan para justificar que las AO no puede estar en las instalaciones del IES: éste ha de obtener buenos resultados de aprendizaje (de "éxito") y un AO contribuiría negativamente (descenderían las puntuaciones medias). Se asume que para mantener un "buen nivel" de resultados, algunos estudiantes han de quedarse fuera y que además de atender la diversidad, el IES tiene que ofrecer una buena imagen que "atraiga" la confianza del alumnado y familias, ofreciéndoles lo que consideran ofertas de calidad (como Bachillerato Bilingüe).

En el discurso de los entrevistados la atención a la diversidad remite a atender a estudiantes con dificultades de aprendizaje, no tanto a aquellos que no se ajustan a las normas, rechazan el entorno escolar, no tienen interés, son problemáticos conductualmente. De hecho, como señala el entrevistado de la Consejería, los Institutos ponen el grito en el cielo cuando se modifica la normativa para exigir como requisito de acceso al AO expediente abierto de absentismo, y priorizar el disciplinario: una muestra de que este alumnado no es bien recibido. Por su parte, entre el profesorado, aunque más o menos maquillada, se argumenta que en los IES la noción de atención a la diversidad mayormente interiorizada es la de ofrecer una enseñanza diferente para aquellos estudiantes más "tontos" y desinteresados. En el AO que acoge los etiquetados de "conflictivos y gente mala" el rechazo a responsabilizarse de su docencia es notable y, en su caso, suele verse "un castigo".

El AO es respaldada solo mientras sea un recurso externo para "liberar" y "descongestionar" las aulas ordinarias "desviando" al alumnado más problemático. Incluso, entre algunos de sus docentes y por algún Departamento de Orientación, se llega a defender que aquellos con un perfil "complejo" no deberían entrar a estas Aulas, quedando fuera del sistema educativo: "son inadaptados, no pueden aprender nada... el fracaso es una opción y hay que aceptarlo".

No sólo se legitima, así, que el AO se localice en instalaciones municipales, separadas del IES, segregando a sus estudiantes, sino también, como se comentará más adelante, se dificulta que regresen y continúen con su escolaridad obligatoria. Una realidad que choca en cierto modo con la "filosofía" oficial de Inclusión abanderada en las normativas y planes formales.

\subsection{LA RESPONSABILIDAD DEL FUNCIONAMIENTO DEL AO RECAE EN SUS DOCENTES}

Las declaraciones de los entrevistados recogidas en la Tabla 4, dejan entrever una asunción de la arraigada distribución del trabajo en las organizaciones escolares: el funcionamiento y resultados del AO dependen fundamentalmente de sus docentes; las funciones de la Administración se limitan a lo burocrático, gestión de recursos, o supervisión formal; los equipos directivos gestionan la puesta en marcha del $\mathrm{AO}$, y mantienen contacto con ella en reuniones esporádicas informativas o para intervenir ante ciertos conflictos o expulsiones; el resto del profesorado no solo no se siente responsable y se queda al margen, sino que en buena medida desconoce qué es el AO; los profesionales "especialistas" de los Departamentos de Orientación, se implican en distinta medida en ocasiones limitándose al PTSC. Predomina el aislamiento y la falta de apoyo en todo lo referido a cuestiones pedagógicas. 
Tabla 4. El AO: un asunto de "sus docentes"

\begin{tabular}{|c|c|}
\hline ADM & $\begin{array}{l}\text { - } \quad \text { Con la Consejería ha desaparecido toda coordinación: la nueva legislación habla } \\
\text { de coordinación institucional, pero no sabemos lo que es. } \\
\text { - La labor del Ayuntamiento con AO, es económica y de cesión de instalaciones ... } \\
\text { el funcionamiento recae básicamente en los profesores, su valor depende de que } \\
\text { tengan una profesionalidad adaptada a esos muchachos. }\end{array}$ \\
\hline ED & $\begin{array}{l}\text { - } \quad \text { Hay muchos cursos y muchas medidas, pero se implican muy pocos. } \\
\text { - } \quad \text { La orientadora ha delegado al PTSC todo lo relativo al AO. } \\
\text { - } \quad \text { Solamente en las evaluaciones, lo obligatorio, ya está, no hay tiempo para } \\
\text { reunirse con el Equipo Docente. } \\
\text { - De vez en cuando tenemos alguna reunión para informar de cuál es la situación y } \\
\text { si ha habido problemas; ese es el seguimiento... no interfiero para nada en esa } \\
\text { dinámica. } \\
\text { - } \\
\text { - Equí se conoce el perfil que va al AO. No, qué se hace allí ni qué se consigue. } \\
\text { Es untigo para sus docentes. }\end{array}$ \\
\hline Docentes & $\begin{array}{l}\text { - } \quad \text { Hay gente que no sabe qué es el AO; si lo sabe, no se presenta } \\
\text { - } \quad \text { En el IES no conozco a nadie ni nada, el profesor a jornada completa no está } \\
\text { integrado. } \\
\text { - } \quad \text { El DO me da orientaciones... pero no hay mucha relación. } \\
\text { - } \quad \text { Falta de apoyo por el Ayuntamiento. }\end{array}$ \\
\hline DO & $\begin{array}{l}\text { - Allí van locos ..., cualquier cosa que falta (tóner, bolsas de basura...); como nexo } \\
\text { - } \quad \text { No el Instituto... colaborar en lo que necesite del Ayuntamiento, esa es mi labor. } \\
\text { menos. Antes tenían una conex con el Aula, antes sí, mucho papeleo... pero este año directa con el centro de origen. } \\
\text { - Para la coordinación hay limitaciones; una descoordinación importante con la } \\
\text { Consejería. }\end{array}$ \\
\hline
\end{tabular}

Fuente. Elaboración propia.

\subsection{UN CURRÍCULO "ALIGERADO EN LO ACADÉMICO"}

Ligado a lo anterior y acorde con esa visión de déficit del alumnado se justifica lo que cabe denominar "rebaje" en el currículo pensado para el AO (Tabla 5).

Tabla 5. Un currículo "para salir del paso"

\begin{tabular}{|l|l|}
\hline ADM & $-\quad \begin{array}{l}\text { Los contenidos son necesarios, pero no el motor del AO (...). Eran dos bloques: uno } \\
\text { para trabajar crecimiento personal, y el académico, obligatorio porque estaban en } \\
\text { edad de escolaridad obligatoria, pero el peso no estaba en lo académico }\end{array}$ \\
& $\begin{array}{l}\text {.. Se pone el foco en los contenidos instrumentales porque es un alumnado que } \\
\text { lo rechaza todo. }\end{array}$ \\
& $\begin{array}{l}\text { Se vincula al perfil profesional de albañilería y pintura porque son trabajos más } \\
\text { manipulativos y menos teóricos que otros perfiles } \\
\text { Su finalidad principal es que aprendan a circular socialmente y que no estén en } \\
\text { casa durmiendo }\end{array}$ \\
\hline
\end{tabular}




\begin{tabular}{|c|c|}
\hline ED & $\begin{array}{l}\text { - .. el hecho en sí mismo de que un alumno lo saques de la calle o no termine a los } \\
\text { dos meses en un programa de Servicios Sociales, pues ya merece la pena (el AO) } \\
\text { - Si un profesor coge los contenidos de } 2^{\circ} \text { de la ESO y pretende darlos, aún con } \\
\text { otra metodología, que se olvide. }\end{array}$ \\
\hline Docentes & $\begin{array}{l}\text { - } \quad \text { Los contenidos) no tienen sentido porque no los van a entender. } \\
\text { - } \quad \text { Los Programas de Trabajo Individualizado: excepción a la regla } \\
\text { - } \quad \text { El que aguanta esa dinámica, asiste, tiene una actitud respetuosa y de atención, } \\
\text { aunque no se entere mucho de los contenidos, está preparado para trabajar. } \\
\text { - Voy más por la vía de que tengan interés en algo ... no estoy en la parte teórica. . }\end{array}$ \\
\hline DO & $\begin{array}{l}\text { - } \quad \text { El AO necesita ir respaldada por una intervención terapéutica } \\
\text { - En el Aula y la FPB el contenido más curricular de Secundaria se lo van a } \\
\text { encontrar... pero no nos engañemos..., es una adaptación grupal..., si no, está } \\
\text { abocado al fracaso; una macro-adaptación curricular en sí misma. }\end{array}$ \\
\hline
\end{tabular}

Fuente. Elaboración propia.

A pesar del diseño oficial, se entiende que, dado el desfase curricular de estudiantes que rechazan "todo" y no se ajustan a las normas, no merece la pena poner el acento en contenidos 'instrumentales', sino, preferentemente en otros ligados al "desarrollo personal". Como señala el entrevistado del Ayuntamiento, los contenidos académicos sólo se tocan "porque" este alumnado está en etapa obligatoria, no porque se valore como relevante una formación rigurosa para todos ellos. Se asume que los más adecuados son los manipulativos, no los "teóricos". Algún entrevistado añade que más importante que la formación que reciban es el que estén en el AO, no en la calle. El sentido educativo de "estar" en el Aula, no se contempla explícitamente.

Entre sus docentes, es prioritario revertir comportamientos, motivar y lograr retener al alumnado. Otorgan menos importancia al currículum oficial, pues se considera inalcanzable dadas las "limitaciones" de este alumnado. Se asume como imprescindible una "macro-adaptación" progresiva: tomar como referencia el currículo de Primaria; eliminar materias "menos útiles" (ciencias) o "más rechazadas por el alumnado" (inglés); obviar Programas de Trabajo Individualizado. Paralelamente, se entiende que mejorar actitudes, comportamientos y nivel de responsabilidad es el mayor reto a alcanzar, siendo suficiente para poder insertarse en el mercado laboral. Lo curricular queda relegado a un segundo plano bajo el supuesto de que ya lo trabajarán si se reincorporan al IES (en particular, a FP Básica) o bien irán aprendiendo "al madurar".

\subsection{UNA EVALUACIÓN "BLANDA”: FAVORECER LA CONTINUIDAD}

Igualmente, la imagen negativa que se tiene del alumnado influye en el reconocimiento de sus resultados en el aula y la facilitación de su continuidad en el sistema escolar. Quienes están directamente ligados a las AO, siempre desde la aludida visión de déficit, entienden que la vía de continuidad de estas Aulas es la FPB, pues si "regresaran al aula ordinaria $\left(4^{\circ}\right.$ ESO) no aprueban ni el recreo". Tal planteamiento se materializa en prácticas de evaluación como: relajar exigencias, redefinir los estándares establecidos, "subir" notas para mejorar la calificación académica (Tabla 6), lo cual se ve refrendado por el hecho de que los docentes del AO priorizan la adquisición de habilidades más que de conocimientos curriculares. 
Tabla 6. Rebajar el rigor de la evaluación y "propiciar continuidad"

\begin{tabular}{|c|c|c|}
\hline ADM & $\begin{array}{l}\text { El planteamiento del AO es que como docente se valore todo lo que va a permitir estar } \\
\text { en FPB, tener éxito. Si tengo que subir la nota, la subo. }\end{array}$ \\
\hline ED & $-\begin{array}{l}\text { Alumnos que no entran a FPB muchas veces se mantienen en los centros... pero } \\
\text { están abocados a la salida inmediata. } \\
\text { Nosotros tenemos la suerte que al ser de informática ... hay cierta selección del } \\
\text { alumnado que viene a FPB. Del AO, ninguno. }\end{array}$ \\
\hline Docentes & $-\begin{array}{l}\text { Hay que seguir, por normativa, unos estándares ... si los seguimos, no aprueban. } \\
\text { Si pertenecemos a Atención a la Diversidad, y valoro el trabajo y evolución de ese } \\
\text { crío, le tengo que poner un notable ino? ... esto es casi una labor social entonces } \\
\text { tenemos que enfocarlo de esa manera... lo otro es independiente, lo irán } \\
\text { aprendiendo con el tiempo. } \\
\text { A FPB podrían salir, pero si los dejas en cuarto normal, no aprueban ni el recreo. }\end{array}$ \\
\hline $\begin{array}{l}\text { La oportunidad del AO es mejorar la calificación académica, uno de los baremos } \\
\text { para entrar a FPB... La norma es el éxito no el fracaso. } \\
\text { Pueden aprovechar para re-engancharse (...) con la FPB porque sabemos que en } \\
\text { la ESO se estrellarían }\end{array}$ \\
\hline
\end{tabular}

Fuente. Elaboración propia.

\subsection{DESVALORIZAR RESULTADOS E "INVISIBILIZAR" AL ALUMANDO}

Por parte de la Administración cabe vislumbrar una cierta reticencia a que los estudiantes del AO accedan a FPB, cuya "buena imagen" quieren proteger. Así lo mostrarían actuaciones declaradas por los entrevistados -desacuerdos entre Direcciones Generales sobre la posibilidad de certificar estas enseñanzas; dificultades para matricularlos en el IES y registrar en sus expedientes las calificaciones obtenidas en el AO; desconfiar de la veracidad de tales calificaciones- (Tabla 7). Son prácticas que, en cierto modo, hacen invisibles a estos estudiantes y dificultan su continuidad. Lo docentes "ajustan" la evaluación para favorecer la continuidad de este alumnado, pero la administración autonómica considera irrelevantes las calificaciones que se les asignan. $\mathrm{El} \mathrm{AO}$, es, en el fondo, una medida terminal: sin posibilidad real de continuar en el sistema escolar. Apenas hay opción para que continúen formándose. En el mejor de los casos, habrá que buscar otras vías. 
Tabla 7. "Invisibilizar" resultados y alumnado

\begin{tabular}{|c|c|}
\hline ED & $\begin{array}{l}\text { - La Administración no ve que esta enseñanza se pueda certificar (...) No hay } \\
\text { acuerdos entre las Direcciones Generales de Ordenación Académica., Formación } \\
\text { Profesional y Atención a la Diversidad, (...) hasta que no ocurra, las AO no } \\
\text { pueden ir bien... } \\
\text { Puedes hacer ahí un esfuerzo humano, tremendo, y seguramente no se va a } \\
\text { reflejar en resultados académicos, nunca. } \\
\text { No existía el sistema informático, ni parámetros... Este año los estudiantes del AO } \\
\text { no he podido matricularlos en mi centro. } \\
\text { Mandamos a los alumnos con calificaciones de 5, 6, 7.... Y, nos llamaron } \\
\text { indignados ... acusándolos que habían falsificado las notas... }\end{array}$ \\
\hline Docentes & $\begin{array}{l}\text { - Los Módulos profesionales no tengo herramientas para certificarlos. } \\
\text { - Se propuso que hiciésemos una evaluación como si fuera de segundo de la ESO, } \\
\text { un paripé... la normativa no dice que la evaluación tiene que ser reglada; en el } \\
\text { Plumier XXI no hay boletín oficial ... ¿qué calificaciones les vamos a poner?, no } \\
\text { sabemos. } \\
\text { El inspector nos va a comunicar qué va a pasar con los alumnos del AO, cómo } \\
\text { vamos a evaluarlos, si se va a hacer un boletín oficial para que se puedan } \\
\text { integrar en la FPB. }\end{array}$ \\
\hline
\end{tabular}

Fuente. Elaboración propia.

\section{CONCLUSIONES}

- La respuesta educativa al alumnado más vulnerable requiere cambios en las formas de hacer y pensar muy arraigadas en las culturas organizativas que pesan como losas sobre estos estudiantes. Ese cambio resulta muy improbable cuando la Administración regula medidas y programas extraordinarios y propicia unas condiciones de implementación que terminan marginando tales programas y, con ello, a su alumnado al que incluso, se le dificultan vías de reenganche y continuidad en su trayectoria escolar.

- La tan preconizada armonización equidad-eficacia desde la esfera político-administrativa choca en la práctica con la presión por los resultados escolares. A éstos no contribuyen los estudiantes con mayores dificultades y peor rendimiento. En base a esto, se legitima un planteamiento de "inclusión excluyente" como el que impregna el AO, que poco contribuye a los cambios en la "gramática básica" que requiere la escuela inclusiva.

- Datos como los presentados ponen de manifiesto que programas o medidas que se articulan como "alternativas" para un alumnado considerado muy problemático, difícilmente podrán ser desplegados adecuadamente como tales si no se promueven las condiciones para desarrollar prácticas que, al tiempo que son alternativas, aseguren una formación académica, social y personal rigurosa y sólida: formación de los profesionales implicados; facilitación de tiempos y posibilidades de coordinación interna del equipo docentes y de éste con el IES, la comunidad y familias, etc.

- El camino hacia una auténtica inclusión educativa es más lento de lo que se proclama. Pese a avances y esfuerzos se establecen distinciones entre quienes merecen estar y permanecen "dentro" del sistema y quienes han de ser relegados a sus márgenes y, a la 
larga, privados de oportunidades y aprendizajes valiosos. La inclusión no se favorece dando por bueno que sea así, o asumiendo que medidas y programas han de implementarse por cumplir con la obligatoriedad de la enseñanza más que por buscar una buena formación y opciones de reenganche educativo.

- Los lenguajes y discursos prevalentes, a todos los niveles, culpan a los sujetos de sus dificultades escolares, no al orden escolar vigente en sus diferentes planos: el sistema educativo, los centros y las aulas se protegen y excusan así, de la necesidad de acometer cambios profundos en su seno que alteren la "cultura" organizativa y escolar en la que se asientan.

- Globalmente considerados, los datos presentados evidencian cómo valores, creencias y rutinas "institucionalizadas" en los centros constituyen una barrera "invisible" importante para posibilitar una práctica escolar y de aula que realmente contribuya al enganche del alumnado con sus aprendizajes y les empuje a persistir e implicarse en su formación escolar. Esto requiere, cuestionar desde dentro modos de pensar y hacer. Seguir investigando y obteniendo conocimiento más profundo sobre temas tan relevantes, será, sin duda, imprescindible.

\section{REFERENCIAS BIBLIOGRÁFICAS}

Arias, F. G. (2012). El Proyecto de Investigación. Introducción a la metodología científica (6. Ed.). Caracas: Editorial Episteme, C. A.

Aristimuño, A. y Parodi, P. (2017). Un Caso Real de Combate al Fracaso en la Educación Pública: Una Cuestión de Acompañamiento, Liderazgo y Cultura Organizacional. Revista Iberoamericana sobre Calidad, Eficacia y Cambio en Educación, 15(4), 141-157.

Atweh B., Bland, D., Carrington, S. y Cavanagh, R. (2007). School Disengagement: Its constructions, investigation and management. AARE 2007. Recuperado de http://www.aare.edu.au/07pap/ atw07598.pdf

Azevedo, J. (2014). O desperdício humano das escolas contamina a democracia. Jornal Público. Recuperado de http://www.joaquimazevedo.com/Images/BibTex/JA_lixo_humano_das_ escolas_Publico_2010.pdf

Bielby, G., Judkins, M., O’Donnell, L. \& McCrone, T. (2012). Review of the Curriculum and Qualification Needs of Young People who are at Risk of Disengagement (NFER Research Programme: From Education to Employment. Slough: NFER.

Brady, P. (2005). Inclusionary and Exclusionary Secondary Schools: The Effect of School Culture on Student Outcomes. Interchange, 36(3), 295-311.

Brown, R. (2004). School Culture and Organization: Lessons from Research and Experience. Recuperado de https://www2.dpsk12.org/pdf/culture_organization.pdf

Clycq, N., Nouwen, W., Van Caudenberg, R., Orozco, M., Van Praag, L. y Timmerman, C. (2017). Theoretical and methodological considerations when studying early school leaving in Europe. CeMIS, University of Antwerp. Recuperado de www.resl-eu.org

Clark, M., Borj, S., Calleja, G., Chircop, F. y Portelli, R. (2005). School attendance Improvement. Malta: Ministry of Education, Youth and Employment. Recuperado de https://www.education. gov.mt/MediaCenter/Docs/

Connolly, M., James, C. \& Beales, B. (2011). Contrasting perspectives on organizational culture change in schools. J. Educational Change, 12, 21-439.

Creswell, J.W. (2015). Research design: Qualitative, Quantitative and mixed approaches (4a Ed.). Thousand Oaks, CA: Sage. 
Dwyer K, Osher D. \& Warger C. (1998). Early warning, timely response: A guide to safe schools. Washington, DC: U.S. Department of Education. Recuperado de https://files.eric.ed.gov/fulltext/ ED418372.pdf

Escudero, J. M. (2016). El fracaso escolar, una forma de exclusión educativa ¿por qué ocurre?, ¿cómo se produce? En J.M. Escudero Muñoz, (comp.) (2016), Inclusión y exclusión educativa: Realidades, miradas y propuestas. (pp. 27-77). Valencia: Nau Llibres.

Escudero, J. M. (coor.) (2013). Estudiantes en riesgo, centros escolares en riesgo. Respuestas educativas al alumnado en situaciones de vulnerabilidad. Murcia: Diego Marín.

Escudero, J. M. y González, M. T. (2013). Estudiantes en riesgo, centros de riesgo: Dimensiones, interpretaciones e implicaciones prácticas. En Escudero, J.M. (Coord.): Estudiantes en riesgo, centros escolares de riesgo: Respuestas educativas al alumnado en situaciones de vulnerabilidad (pp. 13-41). Murcia: Diego Marín.

European Commission (2015). A whole approach to tackling early school leaving Policy messages. European Union. DG Education and Culture.

Ferguson, B., Tilleczek, K., Boydell, K., Rummens, J.A., Cote, D. \& Roth-Edney, D. (2005). Early School Leavers: Understanding the Lived Reality of Student Disengagement from Secondary School. Recuperado de http://www.ontla.on.ca/library/repository/mon/13000/258815.pdf

Friesen, S. (2010, October). Student Engagement, Equity, and the Culture of Schooling. Paper CanadaUnited States Colloquium on Achieving Equity Through Innovation. Toronto. Recuperado de http://cea-ace.s3.amazonaws.com/media/CEA-2010-Colloquium-Friesen.pdf

González, Ma. T. (2003). Cultura y subculturas organizativas. En González, M. T. (Coord.) Organización y gestión de centros escolares: Dimensiones y procesos (pp. 169-186). Madrid: Pearson educación.

. (2005). Conocer y transformar la cultura de las organizaciones educativas. En Santos Guerra, M.A. (Coord.) (2005): Escuelas para la democracia. Cultura, organización y dirección de instituciones educativas (pp.17-40). Santander: Consejería de Educación, Gobierno de Cantabria. . (2015). Los centros escolares y su contribución a paliar el desenganche y abandono escolar. Profesorado, revista de curriculum y formación del profesorado, 19(3), 158-176.

Hernández-Sampieri, R., Fernández, C. y Baptista, P. (2014). Metodología de la investigación ( $6^{a}$ $E d$.) México D.: McGraw Hill Education.

Hynes, M. (2014). Don't call them dropouts: Understanding the experiences of young people who leave high school before graduation. American's Promise Alliance. Recuperado de https://files. eric.ed.gov/fulltext/ED545308.pdf.

Kotter, J. P. (1996). Leading change. Boston: Harvard Business School Press.

L.A.B. (2001). Student-Centered High Schools. Helping schools adapt to the learning needs of adolescents. Recuperado de http://www.alliance.brown.edu/pubs/perspectives/stdntctrhs.pdf

Ladson-Billings, G. (2006). It's not the culture of poverty, it's the poverty of culture: The problem with teacher education. Anthropology \& Education Quarterly, 37(2), 104-109.

López-Yáñez, J. (2004). Cultura organizativa. En F. Salvador, J. L. Rodríguez y A. Bolívar, Diccionario enciclopédico de Didáctica (pp. 263-270). Málaga: Aljibe.

McInerney, P. (2006, November). Blame the student, blame the school or blame the system?': Educational policy and the dilemmas of student engagement and school retention-a Freirean perspective. AARE Annual Conference, Adelaide. Recuperado de https://www.aare.edu.au/data/ publications/2006/mci06168.pdf

McMahon, B., Munns, G., Smyth, J. y Zyngier, D. (2012). Student Engagement for Equity and Social Justice: Creating Space for Student Voice. Teaching \& Learning, 7(2), 63-78.

McMahon, B. \&Zyngier, D. (2009). Student Engagement: Contested concepts in two continents. Research in Comparative and International Education, 4(2).

Miles, M. B., Huberman, A.M. \& Saldaña, J. (2014). Qualitative Data Analysis. A Methods Sourcebook ( $3^{a} E d$.). United States of America: Sage. 
Pinya, C., Pomar, M.I. y Salvà-Mut, F. (2017). Prevenir el abandono educativo en la educación secundaria profesional: aportaciones del alumnado y del profesorado. Profesorado. Revista de currículum y formación del profesorado, 21(4), 95-117.

Russell, V.J., Ainley, M. Frydenberg, E. (2005). Student motivation and engagement. Schooling Issues Digest. Recuperado de https://trove.nla.gov.au/work/153078840?q\&versionId=217780366

Salvá-Mut, FR.; Oliver-Trobat, M. F.; Comas-Forgas, R. (2014). Abandono escolar y desvinculación de la escuela: perspectiva del alumnado. Magis: Revista Internacional de Investigación en Educación, 6(13), 129-142.

Sarasola, M.R. (2004). Una Aproximación al estudio de la cultura organizacional en centros educativos. Archivos Analíticos de Políticas Educativas, 12(57), 1-32

Schein, E. H. (1992). Organizational culture and leadership (2nd ed.). San Francisco: Jossey-Bass.

Shafritz, J. M., \& Ott, J. S. (2001). Organizational culture and sense making. En J. Shafritz \& J. Ott (Eds.), Classics of organization theory (5th ed), (pp. 361-368). San Antonio, TX: Harcourt.

Shields C.M. (2003). Good Intentions Are Not Enough. Transformative Leadership for Communities of Difference. Oxford: Scarecrow Press.

Smyth. J. (2006). "When the students have the power": Students engagement, student voice, and the possibilities for school reform around "dropping out" of school. International Journal of Leadership in education, 9(4), 285-298.

. (2012). When the students "speak back": Student engagement towards a socially just society. In B. McMahon \& J. Portelli (eds), Student engagement in urban school: Beyond neoliberal discourses (pp. 73-90). Charlote, NC: Informate Age Publishing.

Smyth, J. \& Hattam, R. (2002). Early schools leaving and the cultural geography of high schools (1). British Educational Research Journal, 28(3), 375- 397.

Shoda, S. y Guglielmi, S. (2009). A stitch in time: Tackling educational disengagement interim report. Londres: Gotham Rounded.

Tarabini, A. (coord.) (2015). Políticas de lucha contra el abandono escolar en España. Madrid: Síntesis

Taylor, L. \& Parsons, J. (2011). Improving Student Engagement. Current Issues in Education, 14(1). Recuperado de http://cie.asu.edu/

Teddlie, C. \& Tashakkori, A. (2010). Overview of contemporary issues in mixed methods research. In A., Tashakkori, \& C. Teddlie, C. (eds), Handbook of Mixed Methods in Social \& Behavioral Research (pp 1-41). Sage: California.

Thomson, P. y Pennacchia, J. (2016). Hugs and behaviour points: Alternative education and the regulation of 'excluded' youth. International Journal of Inclusive Education, 20(6), 622-640.

Tilleczek, K., Ferguson, B., Roth Edne, D., Rummens, A., Boydell, K. \& Mueller, M. (2011). A Contemporary Study with Early School Leavers: Pathways and Social Processes of Leaving High School. Canadian Journal of Family and Youth, 3(1), 1-39.

Tsang, Kwok Kuen (2009). Three approaches to understanding and investigating the concept of school culture and school culture phenomena: implications to school improvement and school effectiveness. Hong Kong Teachers' Centre Journal, 8, 86-105.

Tyack, L. \& Cuban, L. (2000). En busca de la utopía: un siglo de reformas en las escuelas públicas. México: Fondo de Cultura Económica.

Van Houtte, M. (2005). Climate or culture? A plea for conceptual clarity in school effectiveness research. School effectiveness and school improvement, 16(1), 71-89.

Vázquez Recio, R. (Coord.) (2018). Reconocimiento y bien común en educación. Madrid: Morata

Vázquez-Recio. R. y López-Gil, M. (2018). Interseccionalidad, jóvenes "si sistema" y resistencia. Una mirada diferente del fracaso/ abandono escolar. Revista Brasileira de Educação, 23, 1-23

Vibert, A. B., y Shields, C. (2003). Approaches to student engagement: Does ideology matter? McGill Journal of Education, 32(2), 221-240.

Willms, J. D., Friesen, S. \& Milton, P. (2009). What did you do in school today? Transforming classrooms through social, academic, and intellectual engagement. Toronto: Canadian 
Education Association. Recyoer. Recuperado de http://www.ccl-cca.ca/pdfs/otherreports/ WDYDIST_National_Report_EN.pdf

Wilson, K., Stemp, K. \& McGinty, S. (2011). Re-engaging young people with education and training What are the alternatives? - Youth Studies Australia, 30(4), 32-39.

Zyngier, D. (2005). Doing education not doing time. Engaging pedagogies and pedagogues - what does student engagement look like in action? In P. L. Jeffrey (Ed.), AARE 2004 Melbourne. Doing the Public Good (pp. 2-16). www.aare.edu.au: Australian Association for Research in Education. 
